\title{
Structure-Selectivity Relationships and Structure for a Peptide-Based Enantioselective Acylation Catalyst
}

\author{
Matthew B. Fierman, ${ }^{\ddagger}$ Daniel J. O’Leary, ${ }^{\#}$ Wayne E. Steinmetz ${ }^{\#}$ and Scott J. Miller ${ }^{\ddagger}$ \\ Department of Chemistry, Merkert Chemistry Center, Boston College, Chestnut Hill, \\ Massachusetts 02467-3860 and Department of Chemistry, Pomona College, 645 North \\ College Avenue, Claremont, CA $91711^{\#}$
}

\section{Supporting Information}

\section{General Procedures.}

Proton NMR spectra were recorded on a Varian 400 or $500 \mathrm{MHz}$ spectrometers. Proton chemical shifts are reported in ppm ( $\square$ ) relative to internal tetramethylsilane (TMS, $\square 0.0 \mathrm{ppm}$ ), or with the solvent reference relative to TMS employed as an internal standard $\left(\mathrm{CDCl}_{3}, \square 7.26 \mathrm{ppm}\right)$. Data are reported as follows: chemical shift (multiplicity [singlet (s), doublet (d), triplet (t), quartet (q), multiplet (m)], coupling constants [Hz], integration). All NMR spectra were acquired at ambient temperature. High resolution mass spectra were acquired in the Mass Spectrometry facilities at Boston College (Chestnut Hill, MA.). The method of ionization is indicated in parenthesis.

Analytical GC was performed on a Hewlett-Packard 6890 equipped with a flame ionization detector. HPLC analysis of acylation products were performed using an Hewlett-Packard 1100 employing a diode array detector. Preparative and analytical HPLC performed of peptide catalysts were performed on a Rainin SD-200 chromatograph equipped with a single wavelength UV detector $(214 \mathrm{~nm})$. All peptides were purified by preparative reverse-phase HPLC performed with a X-Terra $\mathrm{RP}_{18} 7 \square \mathrm{m}$ (Waters) column at a flow rate of $5 \mathrm{~mL} / \mathrm{min}$ using a gradient from $60 \%$ water $/ 40 \%$ methanol to $24 \%$ water $/ 76 \%$ methanol over ten minutes, then from $24 \%$ water $/ 76 \%$ methanol to $21 \%$ water $/ 79 \%$ methanol over $5.75 \mathrm{~min}$, from $21 \%$ water $/ 79 \%$ methanol to $0 \%$ water $/ 100 \%$ methanol over $8.5 \mathrm{~min}$. The solvent was held constant at $100 \%$ methanol for 2.5 minutes before returning to $60 \%$ water $/ 40 \%$ methanol over 6 minutes. Peptide purity was examined under similar conditions using an analytical column with flow rate appropriately scaled. Retention times for the peptides are provided below. 
All reactions were performed in oven-dried glassware. All solvents were distilled from appropriate drying agents prior to use. Acetic anhydride was distilled prior to use and was stored in a Schlenk flask. All other reagents were used as received without further purification. In all solid phase syntheses, unless otherwise specified, the term "wash" refers to the following sequence of rinses: $\mathrm{CH}_{2} \mathrm{Cl}_{2}$ (4X), DMF (4X), $\mathrm{CH}_{2} \mathrm{Cl}_{2}$ (4X), DMF (4X).

Selectivity factors. $k_{\text {rel }}$ values were determined using the method of Kagan. $\left[{ }^{1}\right]$ Assays for enantioselectivity and stereochemical proofs were conducted as previously reported. $\left[{ }^{2}\right]$

Solid Phase Peptide Synthesis. Peptides were synthesized on solid support using commercially available HMBA-AM polystyrene resin. $\left[{ }^{3}\right]$ The carboxy terminal amino acid was loaded as the $\mathrm{N}-\mathrm{-}-\mathrm{Fmoc}$ derivative by conventional symmetrical anhydride loading protocols. $\left[{ }^{4}\right]$ A representative procedure (for Peptide $\mathbf{2 b}$ ) is given below:

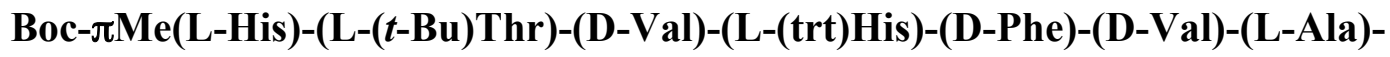
(L-Ile)-OMe (2b). Into a reaction vessel ( $2 \mathrm{ml}$ Bio-Rad chromatography column No. 732-6008) was placed the loaded HMBA-AM resin (0.06 mequiv). To the reaction vessel was added $1.7 \mathrm{ml} 20 \%$ piperidine/ $\mathrm{N}, \mathrm{N}$-dimethylformamide and the reaction was agitated for 30 minutes. The resin was then washed.

To the reaction vessel was then added DMF (0.6 ml), Fmoc-(L-Ala)-OH (79 mg, $0.24 \mathrm{mmol}$ ), HOAt- $\mathrm{H}_{2} \mathrm{O}$ (33 mg, $0.24 \mathrm{mmol}$ ), HBTU (91 mg, $0.24 \mathrm{mmol}$ ), DIPEA (84 $\square \mathrm{L}, 0.48 \mathrm{mmol})$. The reaction mixture was agitated for 3 hours. The resin was then washed.

A $50 \%$ piperidine/DMF solution $(1.7 \mathrm{ml})$ was added to the resin and the mixture was agitated for 7 minutes. The resin was then washed.

To the reaction vessel was then added DMF (0.6 ml), Fmoc-(D-Val)-OH (81 mg, $0.24 \mathrm{mmol}$ ), HOAt- $\mathrm{H}_{2} \mathrm{O}$ (33 mg, $0.24 \mathrm{mmol}$ ), HBTU (91 mg, $0.24 \mathrm{mmol}$ ), DIPEA (84 पL, $0.48 \mathrm{mmol})$. The reaction mixture was agitated for 3 hours. The resin was then washed. 
A 3:1:1 DMF/pyridine/acetic anhydride solution $(1.7 \mathrm{ml})$ was added to the reaction vessel. The mixture was then agitated for 20 minutes. The resin was then washed.

A $20 \%$ piperidine/DMF solution $(1.7 \mathrm{ml})$ was added to the reaction vessel and the mixture was agitated for 30 minutes. The resin was then washed.

To the reaction vessel was then added DMF (0.6 ml), Fmoc-(D-Phe)-OH (93 mg, $0.24 \mathrm{mmol}$ ), HOAt- $\mathrm{H}_{2} \mathrm{O}$ (33 mg, $0.24 \mathrm{mmol}$ ), HBTU (91 mg, $0.24 \mathrm{mmol}$ ), DIPEA (84 $\square \mathrm{L}, 0.48 \mathrm{mmol})$. The reaction mixture was agitated for 3 hours. The resin was then washed.

A $20 \%$ piperidine/DMF solution $(1.7 \mathrm{ml})$ was added to the reaction vessel and the mixture was agitated for 30 minutes. The resin was then washed.

To the reaction vessel was then added DMF (0.6 ml), Fmoc-(L-(trt)His)-OH (149 mg, $0.24 \mathrm{mmol}$ ), HOAt- $\mathrm{H}_{2} \mathrm{O}$ (33 mg, $0.24 \mathrm{mmol}$ ), HBTU (91 mg, $0.24 \mathrm{mmol}$ ), DIPEA $(84 \square \mathrm{L}, 0.48 \mathrm{mmol})$. The reaction mixture was agitated for 3 hours. The resin was then washed.

A $20 \%$ piperidine $/ \mathrm{DMF}$ solution $(1.7 \mathrm{ml})$ was added to the reaction vessel and the mixture was agitated for 30 minutes. The resin was then washed.

To the reaction vessel was then added DMF (0.6 ml), Fmoc-(D-Val)-OH (81 mg, $0.24 \mathrm{mmol}$ ), HOAt- $\mathrm{H}_{2} \mathrm{O}$ (33 mg, $0.24 \mathrm{mmol}$ ), HBTU (91 mg, $0.24 \mathrm{mmol}$ ), DIPEA (84 $\square \mathrm{L}, 0.48 \mathrm{mmol})$. The reaction mixture was agitated for 3 hours. The resin was then washed.

A $20 \%$ piperidine $/ \mathrm{DMF}$ solution $(1.7 \mathrm{ml})$ was added to the reaction vessel and the mixture was agitated for 30 minutes. The resin was then washed.

To the reaction vessel was then added DMF $(0.6 \mathrm{ml})$, Fmoc-(L-( $t$-Bu)Thr)-OH (91 mg, $0.24 \mathrm{mmol}$ ), HOAt- $\mathrm{H}_{2} \mathrm{O}$ (33 mg, $\left.0.24 \mathrm{mmol}\right)$, HBTU (91 mg, $0.24 \mathrm{mmol}$ ), DIPEA ( $84 \square \mathrm{L}, 0.48 \mathrm{mmol})$. The reaction mixture was agitated for 3 hours. The resin was then washed.

A $20 \%$ piperidine/DMF solution $(1.7 \mathrm{ml})$ was added to the reaction vessel and the mixture was agitated for 30 minutes. The resin was then washed.

To the reaction vessel was then added DMF (0.6 ml), BOC- $\square \mathrm{Me}(\mathrm{His})-\mathrm{OH}$ (69 mg, $0.24 \mathrm{mmol}$ ), HOAt- $\mathrm{H}_{2} \mathrm{O}$ (33 mg, $0.24 \mathrm{mmol}$ ), HBTU (91 mg, $0.24 \mathrm{mmol}$ ), DIPEA 
(84 $\square \mathrm{L}, 0.48 \mathrm{mmol}$ ). The reaction mixture was agitated for 3 hours. The resin was then washed. The resin was washed with a $20 \%$ piperidine/DMF solution $(1.7 \mathrm{ml}, 2 \mathrm{X})$, then washed again.

The resin was transferred to a $20 \mathrm{ml}$ Bio-Rad chromatography column. The peptide was cleaved from the resin with a 9:1:1 methanol/DMF/triethylamine $(19 \mathrm{ml})$.

The reaction mixture was agitated for at least 7 days. The cleavage solution was drained and the resin was further washed with $\mathrm{CH}_{2} \mathrm{Cl}_{2}(18 \mathrm{ml}, 2 \mathrm{X})$, methanol (18 $\mathrm{ml}$, $2 \mathrm{X}), \mathrm{CH}_{2} \mathrm{Cl}_{2}(18 \mathrm{ml}, 2 \mathrm{X})$, methanol $(18 \mathrm{ml}, 2 \mathrm{X})$. The cleavage solution and wash solutions were combined and concentrated in vacuo. The resulting yellow residue was purified by preparative reverse-phase HPLC.

Data for Peptides. Characterization for peptide 1 has been previously reported. $\left[^{5}\right]$

Peptide 2b. ${ }^{1} \mathbf{H}$ NMR $\left(\mathrm{CDCl}_{3}, 500 \mathrm{MHz}\right) \square 8.09$ (s, 1H), 7.75 (d, $\left.J=7.5 \mathrm{~Hz}, 1 \mathrm{H}\right), 7.64$ (s, 1H), $7.49(\mathrm{~s}, 1 \mathrm{H}), 7.40-7.22(\mathrm{~m}, 17 \mathrm{H}), 7.20-7.06(\mathrm{~m}, 7 \mathrm{H}), 6.98(\mathrm{~d}, J=9.0 \mathrm{~Hz}, 1 \mathrm{H})$, 6.82, (s, 1H), $6.76(\mathrm{~s}, 1 \mathrm{H}), 6.20,(\mathrm{~s}, 1 \mathrm{H}), 5.51(\mathrm{~d}, J=10.0 \mathrm{~Hz}, 1 \mathrm{H}), 4.63(\mathrm{t}, J=5.0 \mathrm{~Hz}$, $2 \mathrm{H}), 4.58-4.52(\mathrm{~m}, 2 \mathrm{H}), 4.48-4.38(\mathrm{~m}, 2 \mathrm{H}), 4.24(\mathrm{~m}, 2 \mathrm{H}), 4.18(\mathrm{t}, J=5.0 \mathrm{~Hz}, 1 \mathrm{H}), 4.06(\mathrm{t}$, $J=7.0 \mathrm{~Hz}, 1 \mathrm{H}$ ), 3.98 (m, 2H), 3.73 (s, 3H), 3.51 (s, 10H), 3.24-2.84 (m, 7H), 2.19 (m, 1H), $2.08(\mathrm{~m}, 2 \mathrm{H}), 1.90(\mathrm{~m}, 1 \mathrm{H}), 1.59(\mathrm{~s}, 9 \mathrm{H}), 1.43(\mathrm{~s}, 4 \mathrm{H}), 1.40-1.35(\mathrm{~m}, 2 \mathrm{H}), 1.24(\mathrm{~s}$, 4H), $1.02(\mathrm{~d}, J=6.5 \mathrm{~Hz}, 2 \mathrm{H}), 0.98-0.84(\mathrm{~m}, 8 \mathrm{H}), 0.80(\mathrm{~d}, J=6.5 \mathrm{~Hz}, 2 \mathrm{H})$; HPLC

Retention time: $14.2 \mathrm{~min}$; Exact mass calcd for $\left[\mathrm{C}_{74} \mathrm{H}_{100} \mathrm{~N}_{12} \mathrm{O}_{12}+\mathrm{Na}\right]+$ requires $\mathrm{m} / z$ 1371.7495, found: 1371.7481 (ESI +).

Peptide 2a. ${ }^{1} \mathbf{H}$ NMR $\left(\mathrm{CDCl}_{3}, 500 \mathrm{MHz}\right) \square 7.96(\mathrm{~d}, J=8.1 \mathrm{~Hz}, 1 \mathrm{H}), 7.92(\mathrm{~d}, J=5.1 \mathrm{~Hz}$, $1 \mathrm{H}), 7.81(\mathrm{~d}, J=6.6 \mathrm{~Hz}, 1 \mathrm{H}), 7.48(\mathrm{~d}, J=6.6 \mathrm{~Hz}, 1 \mathrm{H}), 7.32-7.30(\mathrm{~m}, 10 \mathrm{H}), 7.14-7.06(\mathrm{~m}$, $13 \mathrm{H}), 6.98(\mathrm{~d}, J=8.1 \mathrm{~Hz}, 1 \mathrm{H}), 6.92(\mathrm{~d}, J=5.9 \mathrm{~Hz}, 1 \mathrm{H}), 6.77(\mathrm{~s}, 1 \mathrm{H}), 6.55(\mathrm{~s}, 1 \mathrm{H}), 5.19$ (d, $J=7.3 \mathrm{~Hz}, 1 \mathrm{H}), 4.58-4.48(\mathrm{~m}, 3 \mathrm{H}), 4.41(\mathrm{t}, J=5.1 \mathrm{~Hz}, 1 \mathrm{H}), 4.33$ (dd, $J=5.9 \mathrm{~Hz}, 2.9$ Hz, 1H), 4.24-4.13 (m, 4H), 4.07 (t, $J=5.9 \mathrm{~Hz}, 1 \mathrm{H}), 3.69$ (s, 3H), 3.47 (s, 3H), 3.10 (dd, $J=13.9 \mathrm{~Hz}, 5.9 \mathrm{~Hz}, 1 \mathrm{H}), 3.00(\mathrm{~d}, J=6.6 \mathrm{~Hz}, 2 \mathrm{H}), 2.90(\mathrm{dd}, J=15.4 \mathrm{~Hz}, 5.9 \mathrm{~Hz}, 1 \mathrm{H})$, $2.81(\mathrm{dd}, J=14.7 \mathrm{~Hz}, 5.1 \mathrm{~Hz}, 1 \mathrm{H}), 2.23-2.15(\mathrm{~m}, 1 \mathrm{H}), 2.11-2.04(\mathrm{~m}, 1 \mathrm{H}), 1.56(\mathrm{~s}, 6 \mathrm{H})$, $1.43(\mathrm{~d}, J=6.6 \mathrm{~Hz}, 3 \mathrm{H}), 1.40(\mathrm{~s}, 9 \mathrm{H}), 1.28(\mathrm{~s}, 9 \mathrm{H}), 1.26(\mathrm{~s}, 9 \mathrm{H}), 1.08(\mathrm{~d}, J=6.6 \mathrm{~Hz}, 3 \mathrm{H})$, 
$0.96(\mathrm{~d}, J=6.6 \mathrm{~Hz}, 3 \mathrm{H}), 0.93(\mathrm{~d}, J=6.6 \mathrm{~Hz}, 3 \mathrm{H}), 0.86(\mathrm{~d}, J=6.6 \mathrm{~Hz}, 3 \mathrm{H}), 0.80(\mathrm{~d}, J=$ $6.6 \mathrm{~Hz}, 3 \mathrm{H}$ ); HPLC Retention time: $21.3 \mathrm{~min}$; Exact mass calcd for $\left[\mathrm{C}_{76} \mathrm{H}_{104} \mathrm{~N}_{12} \mathrm{O}_{13}+\right.$ $\mathrm{Na}]+$ requires $m / z$ 1415.7775, found: $1415.7744(\mathrm{ESI}+$ ).

Peptide 2c. ${ }^{1}$ H NMR $\left(\mathrm{CDCl}_{3}, 500 \mathrm{MHz}\right) \square 7.95(\mathrm{~d}, J=8.8 \mathrm{~Hz}, 1 \mathrm{H}), 7.91(\mathrm{~d}, J=7.3 \mathrm{~Hz}$, 1H), $7.54(\mathrm{~m}, 2 \mathrm{H}), 7.45(\mathrm{~d}, J=5.6 \mathrm{~Hz}, 1 \mathrm{H}), 7.33(\mathrm{~m}, 10 \mathrm{H}), 7.23(\mathrm{~s}, 1 \mathrm{H}), 7.16-7.04(\mathrm{~m}$, 13H), 6.77 (s, 1H), 6.54 (s, 1H), 5.07 (d, $J=8.1 \mathrm{~Hz}, 1 \mathrm{H}), 4.62$ (dd, $J=13.2 \mathrm{~Hz}, 6.6 \mathrm{~Hz}$, $1 \mathrm{H}), 4.49(\mathrm{~m}, 3 \mathrm{H}), 4.40(\mathrm{~m}, 1 \mathrm{H}), 4.20-4.12(\mathrm{~m}, 3 \mathrm{H}), 4.03(\mathrm{~m}, 1 \mathrm{H}), 3.95(\mathrm{~m}, 1 \mathrm{H}), 3.63(\mathrm{~s}$, $3 \mathrm{H}), 3.44(\mathrm{~s}, 3 \mathrm{H}), 3.12(\mathrm{dd}, J=13.9 \mathrm{~Hz}, 5.1 \mathrm{~Hz}, 1 \mathrm{H}), 2.99-2.90(\mathrm{~m}, 4 \mathrm{H}), 2.81(\mathrm{dd}, J=$ $15.4 \mathrm{~Hz}, 7.3 \mathrm{~Hz}, 1 \mathrm{H}), 2.12$ (m, 1H), $1.92(\mathrm{~m}, 1 \mathrm{H}), 1.59$ (br s, 6H), 1.44 (m, 1H), 1.39 (s, 9H), 1.29 (s, 9H), 1.28 (s, 9H), 1.18 (m, 1H), 1.11 (d, $J=6.6 \mathrm{~Hz}, 3 \mathrm{H}), 0.95$ (d, $J=6.6$ $\mathrm{Hz}, 3 \mathrm{H}), 0.91$ (m, 9H); HPLC Retention time: $24.2 \mathrm{~min}$; Exact mass calcd for $\left[\mathrm{C}_{77} \mathrm{H}_{106} \mathrm{~N}_{12} \mathrm{O}_{13}+\mathrm{Na}\right]+$ requires $m / z$ 1429.7906, found: $1429.7900(\mathrm{ESI}+)$.

Peptide 2d. ${ }^{1} \mathrm{H}$ NMR $\left(\mathrm{CDCl}_{3}, 400 \mathrm{MHz}\right) \square 7.98(\mathrm{~d}, J=7.3 \mathrm{~Hz}, 1 \mathrm{H}), 7.87(\mathrm{~d}, J=6.6 \mathrm{~Hz}$, $1 \mathrm{H}), 7.81(\mathrm{~d}, J=8.1 \mathrm{~Hz}, 1 \mathrm{H}), 7.48(\mathrm{~d}, J=5.9 \mathrm{~Hz}, 1 \mathrm{H}), 7.38(\mathrm{~s}, 1 \mathrm{H}), 7.35-7.32(\mathrm{~m}, 10 \mathrm{H})$, 7.11-7.08 (m, 7H), 7.03 (d, $J=6.6 \mathrm{~Hz}, 1 \mathrm{H}), 6.95(\mathrm{~d}, J=7.3 \mathrm{~Hz}, 1 \mathrm{H}), 6.81(\mathrm{~s}, 1 \mathrm{H}), 5.21$ $(\mathrm{d}, J=8.8 \mathrm{~Hz}, 1 \mathrm{H}), 4.67(\mathrm{dd}, J=13.2 \mathrm{~Hz}, 6.6 \mathrm{~Hz}, 1 \mathrm{H}), 4.46(\mathrm{dd}, J=8.1 \mathrm{~Hz}, 4.4 \mathrm{~Hz}, 1 \mathrm{H})$, 4.42-4.24 (m, 4H), 4.20-4.14 (m, 2H), 3.99 (t, $J=6.6 \mathrm{~Hz}, 1 \mathrm{H}), 3.69$ (s, 3H), $3.55(\mathrm{~s}, 3 \mathrm{H})$, 3.08-3.02 (m, 2H), 2.96 (d, $J=6.6 \mathrm{~Hz}, 1 \mathrm{H}), 2.92$ (d, $J=5.9 \mathrm{~Hz}, 1 \mathrm{H}), 2.02-2.07$ (m, 2H), $1.93(\mathrm{~m}, 1 \mathrm{H}), 1.58$ (s, 6H), 1.40 (s, 9H), 1.29 (s, 9H), 1.28 (s, 9H), 1.08 (d, J=5.9 Hz, $3 \mathrm{H}), 0.97-0.90(\mathrm{~m}, 15 \mathrm{H}), 0.86(\mathrm{~d}, J=6.6 \mathrm{~Hz}, 3 \mathrm{H}), 0.83(\mathrm{~d}, J=6.6 \mathrm{~Hz}, 3 \mathrm{H})$; HPLC Retention time: $21.5 \mathrm{~min}$; Exact mass calcd for $\left[\mathrm{C}_{73} \mathrm{H}_{106} \mathrm{~N}_{12} \mathrm{O}_{13}+\mathrm{Na}\right]+$ requires $\mathrm{m} / \mathrm{z}$ 1381.7897, found: 1381.7900 (ESI +).

Peptide 2e. ${ }^{1} \mathbf{H}$ NMR $\left(\mathrm{CDCl}_{3}, 400 \mathrm{MHz}\right) \square 7.96(\mathrm{~d}, J=8.1 \mathrm{~Hz}, 1 \mathrm{H}), 7.52(\mathrm{~m}, 2 \mathrm{H})$, 7.327.15 (m, 8H), 7.04 (d, $J=8.8 \mathrm{~Hz}, 1 \mathrm{H}), 6.94$ (d, $J=5.9 \mathrm{~Hz}, 1 \mathrm{H}), 6.75$ (s, 1H), 5.05 (br s, 1H), 4.60-4.41 (m, 6H), 4.17-4.09 (m, 3H), 3.98 (br s, 1H), $3.70(\mathrm{~s}, 3 \mathrm{H}), 3.40(\mathrm{~s}, 3 \mathrm{H})$, $3.17(\mathrm{dd}, J=13.9 \mathrm{~Hz}, 5.1 \mathrm{~Hz}, 1 \mathrm{H}), 3.06(\mathrm{~m}, 1 \mathrm{H}), 2.95(\mathrm{~d}, J=6.6 \mathrm{~Hz}, 2 \mathrm{H}), 2.27(\mathrm{~m}, 1 \mathrm{H})$, $2.16(\mathrm{~m}, 1 \mathrm{H}), 1.94(\mathrm{~m}, 1 \mathrm{H}), 1.58(\mathrm{~s}, 6 \mathrm{H}), 1.39$ (s, 9H), 1.31 (s, 9H), 1.30 (s, 9H), 1.20 (d, $J=6.6 \mathrm{~Hz}, 3 \mathrm{H}), 1.11(\mathrm{~d}, J=6.6 \mathrm{~Hz}, 3 \mathrm{H}), 1.03-0.92(\mathrm{~m}, 14 \mathrm{H}), 0.86(\mathrm{~d}, J=7.3 \mathrm{~Hz}, 3 \mathrm{H})$; 
HPLC Retention time: 19.6 min; Exact mass calcd for $\left[\mathrm{C}_{56} \mathrm{H}_{95} \mathrm{~N}_{10} \mathrm{O}_{13}\right]+$ requires $\mathrm{m} / \mathrm{z}$ 1127.7086, found: 1127.7080 (ESI +).

Peptide 2f. ${ }^{1} \mathbf{H}$ NMR $\left(\mathrm{CDCl}_{3}, 400 \mathrm{MHz}\right) \square 7.95(\mathrm{~d}, J=6.6 \mathrm{~Hz}, 1 \mathrm{H}), 7.90(\mathrm{~d}, J=7.3 \mathrm{~Hz}$, $1 \mathrm{H}), 7.86(\mathrm{~d}, J=8.1 \mathrm{~Hz}, 1 \mathrm{H}), 7.66(\mathrm{~d}, J=5.9 \mathrm{~Hz}, 1 \mathrm{H}), 7.40-7.29(\mathrm{~m}, 10 \mathrm{H}), 7.17-7.06(\mathrm{~m}$, $14 \mathrm{H}), 6.84(\mathrm{~d}, J=8.1 \mathrm{~Hz}, 1 \mathrm{H}), 6.81(\mathrm{~s}, 1 \mathrm{H}), 6.56(\mathrm{~s}, 1 \mathrm{H}), 5.25(\mathrm{~d}, J=7.3 \mathrm{~Hz}, 1 \mathrm{H}), 4.66-$ $4.10(\mathrm{~m}, 10 \mathrm{H}), 3.69(\mathrm{~s}, 3 \mathrm{H}), 3.51(\mathrm{~s}, 3 \mathrm{H}), .312-2.81(\mathrm{~m}, 6 \mathrm{H}), 2.06(\mathrm{~m}, 1 \mathrm{H}), 1.94(\mathrm{~m}, 1 \mathrm{H})$, 1.74 (br s, 3H), 1.39 (s, 9H), 1.30 (s, 9H), 1.25 (s, 9H), 1.08 (d, $J=6.6 \mathrm{~Hz}, 3 \mathrm{H}), 0.97$ $0.91(\mathrm{~m}, 11 \mathrm{H}), 0.84(\mathrm{~d}, J=7.3 \mathrm{~Hz}, 3 \mathrm{H}), 0.77(\mathrm{~d}, J=6.6 \mathrm{~Hz}, 3 \mathrm{H})$; HPLC Retention time: 26.4 min; Exact mass calcd for $\left[\mathrm{C}_{77} \mathrm{H}_{106} \mathrm{~N}_{12} \mathrm{O}_{13}+\mathrm{Na}\right]+$ requires $\mathrm{m} / z$ 1429.7891, found: $1429.7900(\mathrm{ESI}+)$.

Peptide 2g. ${ }^{1} \mathrm{H}$ NMR $\left(\mathrm{CDCl}_{3}, 500 \mathrm{MHz}\right) \square 7.97(\mathrm{~d}, J=4.4 \mathrm{~Hz}, 1 \mathrm{H}), 7.79(\mathrm{~d}, J=8.1 \mathrm{~Hz}$, $1 \mathrm{H}), 7.35-7.02(\mathrm{~m}, 27 \mathrm{H}), 6.85(\mathrm{~s}, 1 \mathrm{H}), 6.64(\mathrm{~s}, 1 \mathrm{H}), 5.35$ (br s, 1H), $4.50(\mathrm{~m}, 3 \mathrm{H}), 4.38-$ $4.23(\mathrm{~m}, 4 \mathrm{H}), 4.15(\mathrm{t}, J=8.1 \mathrm{~Hz}, 1 \mathrm{H}), 3.95(\mathrm{t}, J=7.3 \mathrm{~Hz}, 1 \mathrm{H}), 3.71(\mathrm{~s}, 3 \mathrm{H}), 3.55(\mathrm{~s}, 3 \mathrm{H})$, 3.34-3.23 (m, 2H), 3.05-2.93 (m, 3H), 2.72 (dd, $J=14.6 \mathrm{~Hz}, 8.8 \mathrm{~Hz}, 1 \mathrm{H}), 2.20$ (m, 1H), $2.06(\mathrm{~m}, 1 \mathrm{H}), 1.96(\mathrm{~m}, 1 \mathrm{H}), 1.57$ (br s, 3H), $1.43(\mathrm{~s}, 9 \mathrm{H}), 1.27$ (s, 9H), 1.23 (d, $J=7.3 \mathrm{~Hz}$, $3 \mathrm{H}), 1.13(\mathrm{~d}, J=5.9 \mathrm{~Hz}, 3 \mathrm{H}), 0.97-0.91(\mathrm{~m}, 14 \mathrm{H}), 0.88$ (d, $J=6.6 \mathrm{~Hz}, 3 \mathrm{H}), 0.84(\mathrm{~d}, J=$ $6.6 \mathrm{~Hz}, 3 \mathrm{H})$; HPLC Retention time: $24.0 \mathrm{~min}$; Exact mass calcld for $\left[\mathrm{C}_{74} \mathrm{H}_{100} \mathrm{~N}_{12} \mathrm{O}_{12}+\right.$ $\mathrm{Na}]+$ requires $m / z$ 1371.7494, found: $1371.7481(\mathrm{ESI}+$ ).

Peptide 2h. ${ }^{1}$ H NMR $\left(\mathrm{CDCl}_{3}, 400 \mathrm{MHz}\right) \square 7.96(\mathrm{~d}, J=5.9 \mathrm{~Hz}, 1 \mathrm{H}), 7.84(\mathrm{~m}, 2 \mathrm{H}), 7.54$ (d, $J=6.6 \mathrm{~Hz}, 1 \mathrm{H}), 7.34-7.31(\mathrm{~m}, 8 \mathrm{H}), 7.17-6.98(\mathrm{~m}, 15 \mathrm{H}), 6.73(\mathrm{~d}, J=7.3 \mathrm{~Hz}, 1 \mathrm{H}), 6.54$ (s, 1H), 5.09 (d, $J=8.1 \mathrm{~Hz}, 1 \mathrm{H}), 4.61(\mathrm{dd}, J=13.9 \mathrm{~Hz}, 7.3 \mathrm{~Hz}, 1 \mathrm{H}), 4.50(\mathrm{~m}, 2 \mathrm{H}), 4.40$ (m, 2H), 4.27-4.07 (m, 5H), 3.69 (s, 3H), 3.11 (dd, $J=13.2 \mathrm{~Hz}, 6.6 \mathrm{~Hz}, 1 \mathrm{H}), 3.99-2.88$ (m, 2H), 2.43 (dd, $J=16.1 \mathrm{~Hz}, 6.6 \mathrm{~Hz}, 1 \mathrm{H}), 2.20$ (m, 1H), 2.07 (m, 1H), $1.94(\mathrm{~m}, 1 \mathrm{H})$, 1.58 (br s, 6H), 1.42 (s, 9H), 1.29 (s, 9H), 1.28 (s, 9H), 1.23 (d, $J=6.6 \mathrm{~Hz}, 3 \mathrm{H}), 1.07$ (d, $J=5.9 \mathrm{~Hz}, 3 \mathrm{H}), 0.99$ (d, $J=6.6 \mathrm{~Hz}, 3 \mathrm{H}), 0.93(\mathrm{~m}, 8 \mathrm{H}), 0.83$ (d, $J=6.6 \mathrm{~Hz}), 0.78$ (d, $J=$ $7.3 \mathrm{~Hz}, 3 \mathrm{H}$ ); HPLC Retention time: $22.5 \mathrm{~min}$; Exact mass calcd for $\left[\mathrm{C}_{76} \mathrm{H}_{104} \mathrm{~N}_{12} \mathrm{O}_{13}+\right.$ $\mathrm{Na}]+$ requires $m / z$ 1415.7775, found: $1415.7744(\mathrm{ESI}+)$. 
Peptide 5. ${ }^{1} \mathrm{H}$ NMR (CDCl3, $\left.500 \mathrm{MHz}\right): \square 8.13(\mathrm{~d}, J=11.1 \mathrm{~Hz}, 1 \mathrm{H}), 8.12(\mathrm{~d}, J=11.0$ $\mathrm{Hz}, 1 \mathrm{H}), 7.79(\mathrm{~d}, J=8.1 \mathrm{~Hz}, 1 \mathrm{H}), 7.57(\mathrm{~d}, J=7.3 \mathrm{~Hz}, 1 \mathrm{H}), 7.42(\mathrm{~d}, J=8.1 \mathrm{~Hz}, 1 \mathrm{H}), 7.37-$ 6.99(m, 22H), 6.77(s, 1H), 6.64(s, 1H), 5.01(s, 1H), 4.66(dd, $J=13.9 \mathrm{~Hz}, 6.6 \mathrm{~Hz}, 1 \mathrm{H})$, $4.51-4.47(\mathrm{~m}, 2 \mathrm{H}), 4.42-4.40(\mathrm{~m}, 2 \mathrm{H}), 4.28(\mathrm{t}, J=5.9 \mathrm{~Hz}, 1 \mathrm{H}), 4.24-4.10(\mathrm{~m}, 5 \mathrm{H}), 3.71(\mathrm{~s}$, $3 \mathrm{H}), 3.50(\mathrm{~s}, 3 \mathrm{H}), 3.08-2.85(\mathrm{~m}, 7 \mathrm{H}), 2.26(\mathrm{dd}, J=11.7 \mathrm{~Hz}, 6.6 \mathrm{~Hz}), 2.13(\mathrm{~m}, 1 \mathrm{H}), 1.93(\mathrm{~m}$, $1 \mathrm{H}), 1.58(\mathrm{~s}, 8 \mathrm{H}), 1.39(2,8 \mathrm{H}), 1.27(\mathrm{~d}, J=11.0 \mathrm{~Hz}, 16 \mathrm{H}), 1.08(\mathrm{~d}, J=6.6 \mathrm{~Hz}, 3 \mathrm{H}), 0.98-$ $0.85(\mathrm{~m}, 16 \mathrm{H}), 0.78(\mathrm{~d}, J=6.6 \mathrm{~Hz}, 3 \mathrm{H})$; HPLC Retention time $23.7 \mathrm{~min}$; Exact mass calcd for $\left[\mathrm{C}_{79} \mathrm{H}_{110} \mathrm{~N}_{12} \mathrm{O}_{13}+\mathrm{Na}\right]+$ requires $m / z$ 1457.8210, found: $1457.8213($ ESI + ).

Peptide 6. ${ }^{1} \mathbf{H}$ NMR $\left(\mathrm{CDCl}_{3}, 500 \mathrm{MHz}\right) \square 8.09(\mathrm{~d}, J=7.3 \mathrm{~Hz}, 1 \mathrm{H}), 7.96(\mathrm{~d}, J=5.1 \mathrm{~Hz}$, $1 \mathrm{H}), 7.52(\mathrm{~d}, J=6.6 \mathrm{~Hz}, 1 \mathrm{H}), 7.35-7.33(\mathrm{~m}, 10 \mathrm{H}), 7.20-7.07(\mathrm{~m}, 11 \mathrm{H}), 6.92(\mathrm{~d}, J=8.1 \mathrm{~Hz}$, $1 \mathrm{H}), 6.81(\mathrm{~s}, 1 \mathrm{H}), 6.66(\mathrm{~d}, J=8.8 \mathrm{~Hz}, 1 \mathrm{H}), 6.58(\mathrm{~s}, 1 \mathrm{H}), 5.22(\mathrm{~d}, J=8.1 \mathrm{~Hz}, 1 \mathrm{H}), 4.61(\mathrm{p}, J$ $=7.3 \mathrm{~Hz}, 2 \mathrm{H}), 4.50(\mathrm{dd}, J=9.5 \mathrm{~Hz}, 2.2 \mathrm{~Hz}, 1 \mathrm{H}), 4.45(\mathrm{t}, J=5.1 \mathrm{~Hz}, 1 \mathrm{H}), 4.30-4.13(\mathrm{~m}$, $5 \mathrm{H}), 3.72(\mathrm{~s}, 3 \mathrm{H}), 3,50(\mathrm{~s}, 3 \mathrm{H}), 3.12(\mathrm{dd}, J=13.9 \mathrm{~Hz}, 5.9 \mathrm{~Hz}, 1 \mathrm{H}), 3.04-2.96(\mathrm{~m}, 3 \mathrm{H})$, $2.80(\mathrm{dd}, J=15.4 \mathrm{~Hz}, 6.6 \mathrm{~Hz}, 1 \mathrm{H}), 2.24(\mathrm{ddd}, J=14.7 \mathrm{~Hz}, 14.7 \mathrm{~Hz}, 8.1 \mathrm{~Hz}, 1 \mathrm{H}), 2.12(\mathrm{ddd}$, $J=13.2 \mathrm{~Hz}, 13.2 \mathrm{~Hz}, 6.6 \mathrm{~Hz}, 1 \mathrm{H}), 1.62(\mathrm{~s}, 3 \mathrm{H}), 1.42(\mathrm{~s}, 9 \mathrm{H}), 1.29(\mathrm{~s}, 9 \mathrm{H}), 1.15(\mathrm{~d}, J=6.6$ $\mathrm{Hz}, 3 \mathrm{H}), 0.99-0.94(\mathrm{~m}, 9 \mathrm{H}), 0.87(\mathrm{~d}, J=7.3 \mathrm{~Hz}, 3 \mathrm{H}), 0.82(\mathrm{~d}, J=6.6 \mathrm{~Hz}, 3 \mathrm{H})$; HPLC Retention time: $23.5 \mathrm{~min}$; Exact mass calcd for $\left[\mathrm{C}_{73} \mathrm{H}_{99} \mathrm{~N}_{11} \mathrm{O}_{12}+\mathrm{Na}\right]+$ requires $\mathrm{m} / \mathrm{z}$ 1344.7383, found: 1344.7372 (ESI +).

Peptide 7. ${ }^{1} \mathbf{H}$ NMR $\left(\mathrm{CDCl}_{3}, 500 \mathrm{MHz}\right): \square 8.04(\mathrm{~d}, J=8.1 \mathrm{~Hz}, 1 \mathrm{H}), 7.99(\mathrm{~d}, J=5.9 \mathrm{~Hz}$, $1 \mathrm{H}), 7.49(\mathrm{~d}, J=5.9 \mathrm{~Hz}, 1 \mathrm{H}), 7.35-7.32(\mathrm{~m}, 10 \mathrm{H}), 7.19-7.07(\mathrm{~m}, 11 \mathrm{H}), 6.79(\mathrm{~m}, 2 \mathrm{H})$, $6.57(\mathrm{~s}, 1 \mathrm{H}), 5.22(\mathrm{~d}, J=6.6 \mathrm{~Hz}, 1 \mathrm{H}), 4.56(\mathrm{~m}, 2 \mathrm{H}), 4.44(\mathrm{t}, J=4.4 \mathrm{~Hz}, 1 \mathrm{H}), 4.40(\mathrm{dd}, J=$ $8.8 \mathrm{~Hz}, 5.9 \mathrm{~Hz}, 1 \mathrm{H}), 4.19(\mathrm{~m}, 2 \mathrm{H}), 4.11(\mathrm{t}, J=6.6 \mathrm{~Hz}, 1 \mathrm{H}), 3.67(\mathrm{~s}, 3 \mathrm{H}), 3.51(\mathrm{~s}, 3 \mathrm{H})$, $3.14(\mathrm{dd}, J=13.9 \mathrm{~Hz}, 6.6 \mathrm{~Hz}, 1 \mathrm{H}), 3.03-2.94(\mathrm{~m}, 4 \mathrm{H}), 2.82(\mathrm{dd}, J=15.4 \mathrm{~Hz}, 5.9 \mathrm{~Hz}, 1 \mathrm{H})$, 2.22(ddd, $J=13.2 \mathrm{~Hz}, 13.2 \mathrm{~Hz}, 6.6 \mathrm{~Hz}, 1 \mathrm{H}$ ), $2.05(\mathrm{ddd}, J=13.9 \mathrm{~Hz}, 13.9 \mathrm{~Hz}, 6.6 \mathrm{~Hz}$, $1 \mathrm{H}), 1.61(\mathrm{~s}, 3 \mathrm{H}), 1.42(\mathrm{~s}, 9 \mathrm{H}), 1.30(\mathrm{~s}, 9 \mathrm{H}), 0.97(\mathrm{~m}, 9 \mathrm{H}), 0.84(\mathrm{~d}, J=7.3 \mathrm{~Hz}, 3 \mathrm{H}), 0.80(\mathrm{~d}, J$ $=6.6 \mathrm{~Hz}, 3 \mathrm{H}$ ); HPLC Retention time: $19.1 \mathrm{~min}$; Exact mass calcd for $\left[\mathrm{C}_{65} \mathrm{H}_{84} \mathrm{~N}_{10} \mathrm{O}_{10}+\right.$ $\mathrm{Na}]+$ requires $m / z$ 1187.6270, found: $1187.6270(\mathrm{ESI}+)$. 
Peptide 8. ${ }^{1} \mathbf{H}$ NMR $\left(\mathrm{CDCl}_{3}, 500 \mathrm{MHz}\right)$ : $\square 8.38(\mathrm{~d}, J=7.3 \mathrm{~Hz}, 1 \mathrm{H}), 7.65(\mathrm{~d}, J=8.1 \mathrm{~Hz}$, $1 \mathrm{H}), 7.53(\mathrm{~d}, J=7.3 \mathrm{~Hz}, 1 \mathrm{H}), 7.36-7.32(\mathrm{~m}, 9 \mathrm{H}), 7.22-7.07(\mathrm{~m}, 11 \mathrm{H}), 6.78(\mathrm{~s}, 1 \mathrm{H}), 6.63(\mathrm{~s}$, $1 \mathrm{H}), 5.05(\mathrm{~d}, J=6.6 \mathrm{~Hz}, 1 \mathrm{H}), 4.71(\mathrm{p}, J=6.6 \mathrm{~Hz}, 2 \mathrm{H}), 4.46(\mathrm{t}, J=5.1 \mathrm{~Hz}, 1 \mathrm{H}), 4.23-$ $4.16(\mathrm{~m}, 3 \mathrm{H}), 3.60(\mathrm{~s}, 3 \mathrm{H}), 3.49(\mathrm{~s}, 3 \mathrm{H}), 3.14-3.07(\mathrm{~m}, 2 \mathrm{H}), 3.02-2.97(\mathrm{~m}, 3 \mathrm{H}), 2.80(\mathrm{dd}, J=$ $15.4 \mathrm{~Hz}, 5.9 \mathrm{~Hz}, 1 \mathrm{H}), 2.27(\mathrm{ddd}, J=13.2 \mathrm{~Hz}, 13.2 \mathrm{~Hz}, 6.6 \mathrm{~Hz}, 1 \mathrm{H}), 1.63(\mathrm{~s}, 3 \mathrm{H}), 1.42(\mathrm{~s}$, $9 \mathrm{H}), 1.29(\mathrm{~s}, 9 \mathrm{H}), 1.00(\mathrm{~d}, J=6.6 \mathrm{~Hz}, 3 \mathrm{H}), 0.99(\mathrm{~d}, J=5.9 \mathrm{~Hz}, 3 \mathrm{H}), 0.95(\mathrm{~d}, J=6.6 \mathrm{~Hz}$, $3 \mathrm{H}), 0.88(\mathrm{~d}, J=6.6 \mathrm{~Hz}, 3 \mathrm{H})$; HPLC Retention time: $17.2 \mathrm{~min}$; Exact mass calcd for $\left[\mathrm{C}_{60} \mathrm{H}_{75} \mathrm{~N}_{9} \mathrm{O}_{9}+\mathrm{Na}\right]+$ requires $m / z$ 1088.5574, found: $1088.5585(\mathrm{ESI}+)$.

Peptide 9. ${ }^{1} \mathbf{H}$ NMR $\left(\mathrm{CDCl}_{3}, 500 \mathrm{MHz}\right)$ : observed as two major conformations $\square 8.15$ $(\mathrm{d}, J=7.3 \mathrm{~Hz}, 1 \mathrm{H}), 7.92$ (d, $J=5.1 \mathrm{~Hz}, 1 \mathrm{H}), 7.58$ (d, $J=9.5 \mathrm{~Hz}, 1 \mathrm{H}), 7.56$ (d, $J=8.1 \mathrm{~Hz}$, $1 \mathrm{H}), 7.37-7.05(\mathrm{~m}, 16 \mathrm{H}), 6.83(\mathrm{~s}, 0.5 \mathrm{H}), 6.79(\mathrm{~s}, 0.5 \mathrm{H}), 6.54(\mathrm{~s}, 0.5 \mathrm{H}), 6.52(\mathrm{~s}, 0.5 \mathrm{H})$, $5.03(\mathrm{~m}, 1 \mathrm{H}), 4.80(\mathrm{~m}, 1 \mathrm{H}), 4.51(\mathrm{t}, J=4.4 \mathrm{~Hz}, 0.5 \mathrm{H}), 4.43(\mathrm{~m}, 1 \mathrm{H}), 4.38$ (t, $J=4.4 \mathrm{~Hz}$, $0.5 \mathrm{H}), 4.27(\mathrm{~m}, 1 \mathrm{H}), 4.16(\mathrm{~m}, 1 \mathrm{H}), 3.57(\mathrm{~s}, 1.5 \mathrm{H}), 3.56(\mathrm{~s}, 1.5 \mathrm{H}), 3.54(\mathrm{~s}, 1.5 \mathrm{H}), 3.52(\mathrm{~s}$, $1.5 \mathrm{H}), 3.10-2.88(\mathrm{~m}, 4 \mathrm{H}), 2.37(\mathrm{~m}, 0.5 \mathrm{H}), 2.24(\mathrm{~m}, 0.5 \mathrm{H}), 1.41(\mathrm{~s}, 9 \mathrm{H}), 1.28(\mathrm{~s}, 4.5 \mathrm{H})$, 1.24 (s, 4.5H), 1.01-0.93 (m, 9H); HPLC Retention time: $13.1 \mathrm{~min}$; Exact mass calcd for $\left[\mathrm{C}_{51} \mathrm{H}_{66} \mathrm{~N}_{8} \mathrm{O}_{8}+\mathrm{Na}\right]+$ requires $m / z$ 941.4908, found: $941.4901(\mathrm{ESI}+$ ).

\section{General procedure for kinetic resolution of secondary alcohols.}

To a $10 \mathrm{~mL}$ round bottom flask equipped with a stir bar was added peptide $(0.001$ mmol, dissolved in $100 \square \mathrm{L}$ toluene), alcohol (400 $\square \mathrm{L}$ of a $0.5 \mathrm{M}$ stock solution in toluene, $0.200 \mathrm{mmol})$, Hunig's base $(14 \square \mathrm{L}, 0.080 \mathrm{mmol})$, and toluene $(1 \mathrm{~mL})$. The resulting solution and a stock solution of acetic anhydride (0.6 M, in toluene) were cooled to $-78{ }^{\circ} \mathrm{C}$. The acetic anhydride solution $(500 \square \mathrm{L}, 0.300 \mathrm{mmol})$ was added to the flask. The flask was placed in a cryocooled bath set to $-65^{\circ} \mathrm{C}$. The reaction was stirred for 12 h. The reactions were quenched with $1.0 \mathrm{~mL}$ of methanol. Proofs of stereochemistry and descriptions of enantioselectivity assays for all substrates and products have been described previously. $\left[{ }^{6}\right]$ 


\section{Solution-Phase Structural Studies.}

General Procedures. All NMR spectra used for proton assignment and to acquire 2D ROESY data for peptide 1 was acquired on a Bruker DPX $400 \mathrm{MHz}$ NMR spectrometer. This data was processed using XWINNMR 2.6. All GOESYTR data was acquired on a Varian Unity-Inova $500 \mathrm{MHz}$ NMR spectrometer. Varian data was processed using VNMR 6.1 B.

Table S1. ${ }^{1} \mathrm{H}$ NMR data for peptide 1. All atoms are labeled from the $\square \square$ carbon along

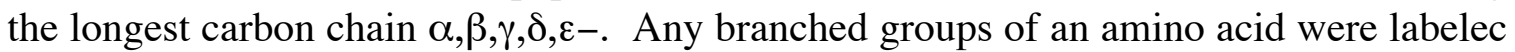
sequentially after the last carbon on the longest chain. In the case of nonequivalent, diastereotopic protons, the higher field proton was labeled with a prime (').

\begin{tabular}{|c|c|c|}
\hline$\underline{\text { Residue }}$ & Proton & ㄱ-(ppm) \\
\hline BOC & $-\mathrm{CH}_{3}$ & 1.41 \\
\hline \multirow[t]{6}{*}{ L-PMH1 } & $\mathrm{NH}\left(\mathrm{J}_{\mathrm{NHD}}=8.1 \mathrm{~Hz}\right)$ & 5.22 \\
\hline & $\square \mathrm{H}$ (overlap with Val6 $\square \mathrm{H}$ ) & 4.25 \\
\hline & $\square \mathrm{H}, \square^{\prime} \mathrm{H}(\mathrm{J}=5.6 \mathrm{~Hz})$ & 3.00 \\
\hline & Imid $2 \mathrm{CH}$ (overlap with ArH) & 7.35 \\
\hline & Imid $5 \mathrm{CH}$ & 6.78 \\
\hline & $\pi-\mathrm{CH}_{3}$ & 3.45 \\
\hline \multirow[t]{5}{*}{ L-( $t$-Bu)THR2 } & $\mathrm{NH}\left(\mathrm{J}_{\mathrm{NHD}}=3.0 \mathrm{~Hz}\right)$ & 7.19 \\
\hline & $\square \mathrm{H}$ (overlap with Thr $\square \mathrm{H7}$ ) & 4.40 \\
\hline & $\square \mathrm{H}$ (overlap with Thr $\square \mathrm{H} 7$ ) & 4.19 \\
\hline & {$\left[\mathrm{CH}_{3}\left(\mathrm{~J}_{\square}=6.6 \mathrm{~Hz}\right)\right.$} & 0.98 \\
\hline & $\mathrm{CH}_{3}$ & 1.30 \\
\hline \multirow[t]{4}{*}{ D-VAL3 } & $\mathrm{NH}\left(\mathrm{J}_{\mathrm{NH} \square}=6.6 \mathrm{~Hz}\right)$ & 7.51 \\
\hline & $\square \mathrm{H}(\mathrm{J}=6.1 \mathrm{~Hz})$ & 4.09 \\
\hline & $\square \mathrm{H}$ & 2.19 \\
\hline & {$\left[\mathrm{CH}_{3}, \mathrm{CCH}_{3}(\mathrm{~J}=7.1 \mathrm{~Hz})\right.$} & 0.94 \\
\hline \multirow[t]{7}{*}{ L-([-trt)HIS4 } & $\mathrm{NH}\left(\mathrm{J}_{\mathrm{NH} \mathrm{D}}=6.6 \mathrm{~Hz}\right)$ & 8.01 \\
\hline & $\square \mathrm{H}(\mathrm{J}=13.1,6.6 \mathrm{~Hz})$ & 4.61 \\
\hline & $\square \mathrm{H}\left(\mathrm{J}_{\square \square}=15.2 \mathrm{~Hz}, \mathrm{~J}_{\square \square}=5.6 \mathrm{~Hz}\right)$ & 2.92 \\
\hline & $\square^{\prime} \mathrm{H}\left(\mathrm{J}_{\square}=15.2 \mathrm{~Hz}, \mathrm{~J}_{\square}=6.1 \mathrm{~Hz}\right)$ & 2.84 \\
\hline & Imid $2 \mathrm{CH}$ (overlap with ArH) & 7.10 \\
\hline & Imid $5 \mathrm{CH}$ & 6.57 \\
\hline & ArH (2 multiplets) & $7.35,7.10$ \\
\hline \multirow[t]{3}{*}{ D-PHE5 } & $\mathrm{NH}\left(\mathrm{J}_{\mathrm{NH} \mathrm{D}}=6.6 \mathrm{~Hz}\right)$ & 7.98 \\
\hline & $\square \mathrm{H}(\mathrm{J}=14.1,8.7 \mathrm{~Hz})$ & 4.57 \\
\hline & $\square \mathrm{H}\left(\mathrm{J}_{\square \square}=13.9 \mathrm{~Hz}, \mathrm{~J}_{\square \square}=5.7 \mathrm{~Hz}\right)$ & 3.08 \\
\hline
\end{tabular}




\begin{tabular}{|c|c|c|}
\hline & $\square^{\prime} \mathrm{H}\left(\mathrm{J}_{\square \square}=12.6 \mathrm{~Hz}, \mathrm{~J}_{\square \square}=5.7 \mathrm{~Hz}\right)$ & 2.98 \\
\hline & ArH (2 multiplets) & $7.35,7.10$ \\
\hline \multirow[t]{5}{*}{ D-VAL6 } & $\mathrm{NH}\left(\mathrm{J}_{\mathrm{NHD}}=6.1 \mathrm{~Hz}\right)$ & 7.03 \\
\hline & $\square \mathrm{H}$ (overlaps with PMH1) & 4.24 \\
\hline & $\square \mathrm{H}$ & 2.13 \\
\hline & $\mathrm{CCH}_{3}(\mathrm{~J}=6.6 \mathrm{~Hz})$ & 0.85 \\
\hline & $\mathrm{CCH}_{3}(\mathrm{~J}=6.6 \mathrm{~Hz})$ & 0.84 \\
\hline \multirow[t]{5}{*}{$\mathrm{L}-(t-\mathrm{Bu}) \mathrm{THR} 7$} & $\mathrm{NH}\left(\mathrm{J}_{\mathrm{NH}}=3.5 \mathrm{~Hz}\right)$ & 7.04 \\
\hline & \H (overlap with Thr $\square \mathrm{H} 2$ ) & 4.41 \\
\hline & $\square \mathrm{H}$ (overlap with $\mathrm{Thr} \square \mathrm{H} 2$ ) & 4.17 \\
\hline & $\mathrm{CH}_{3}\left(\mathrm{~J}_{\square}=6.6 \mathrm{~Hz}\right)$ & 1.09 \\
\hline & $\mathrm{CH}_{3}$ & 1.31 \\
\hline \multirow[t]{6}{*}{ L-ILE8 } & $\mathrm{NH}\left(\mathrm{J}_{\mathrm{NH}}=8.1 \mathrm{~Hz}\right)$ & 7.84 \\
\hline & $\square \mathrm{H}(\mathrm{J}=8.2,4.7 \mathrm{~Hz})$ & 4.49 \\
\hline & $\square \mathrm{H}$ & 1.94 \\
\hline & $\mathrm{CH}$ (overlap with $\mathrm{BOC} \mathrm{CH}_{3}$ ) & 1.47 \\
\hline & $\overline{\mathrm{DH}}$ (overlap with $\mathrm{Thr} 2 \mathrm{CH}_{3}$ and $\mathrm{Thr} 7 \mathrm{CH}_{3}$ & 1.23 \\
\hline & $\square \mathrm{H}, \square \mathrm{H}(\mathrm{J}=5.1 \mathrm{~Hz})$ & 0.95 \\
\hline Methyl Ester (OMe) & $\mathrm{CH}_{3}$ & 3.71 \\
\hline
\end{tabular}

Table S2. Interproton distance restraints for peptide 1. Restraints added for final round calculation are shaded in grey.

\begin{tabular}{|c|c|}
\hline Proton Pair & Upper Bound $(\AA)$ \\
\hline РMH1C, РMH1С $\pi$ & 5 \\
\hline HIS4NH, VAL3ロH & 3 \\
\hline PHE5NH, VAL6NH & 4 \\
\hline VAL3NH, HIS4NH & 4 \\
\hline PMH1NH, THR2NH & 4 \\
\hline PMH1NH, PMH1Imid5H & 4 \\
\hline THR2NH, PMH1 $\square \mathrm{H}$ & 3 \\
\hline VAL3NH, THR2 $\square \mathrm{H}$ & 3 \\
\hline ILE8NH, THR7ロH & 4 \\
\hline VAL6NH, PHE5 $\square \mathrm{H}$ & 3 \\
\hline PMH1C, THR2NH & 5 \\
\hline THR7CD, ILE8NH & 5 \\
\hline THR7CD, OMeC & 6 \\
\hline THR7CD, VAL6NH & 5 \\
\hline PMH1C, THR7 $\square \mathrm{H}$ & 5 \\
\hline VAL6C $\square$ THR7ПH & 5 \\
\hline VAL6CD, THR7ПH & 5 \\
\hline HIS4NH, VAL3ロH & 4 \\
\hline
\end{tabular}




\begin{tabular}{|c|c|}
\hline 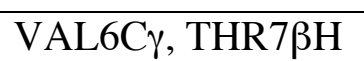 & 5 \\
\hline VAL6C $\square$, THR7ПH & 5 \\
\hline THR7NH, ILE8NH & 4 \\
\hline ILE8NH, THR7ロH & 4 \\
\hline THR7NH, THR7】H & 4 \\
\hline PMH1C】, ILE8NH & 5 \\
\hline PMH1C $\pi$, THR2 $\square \mathrm{H}$ & 5 \\
\hline
\end{tabular}




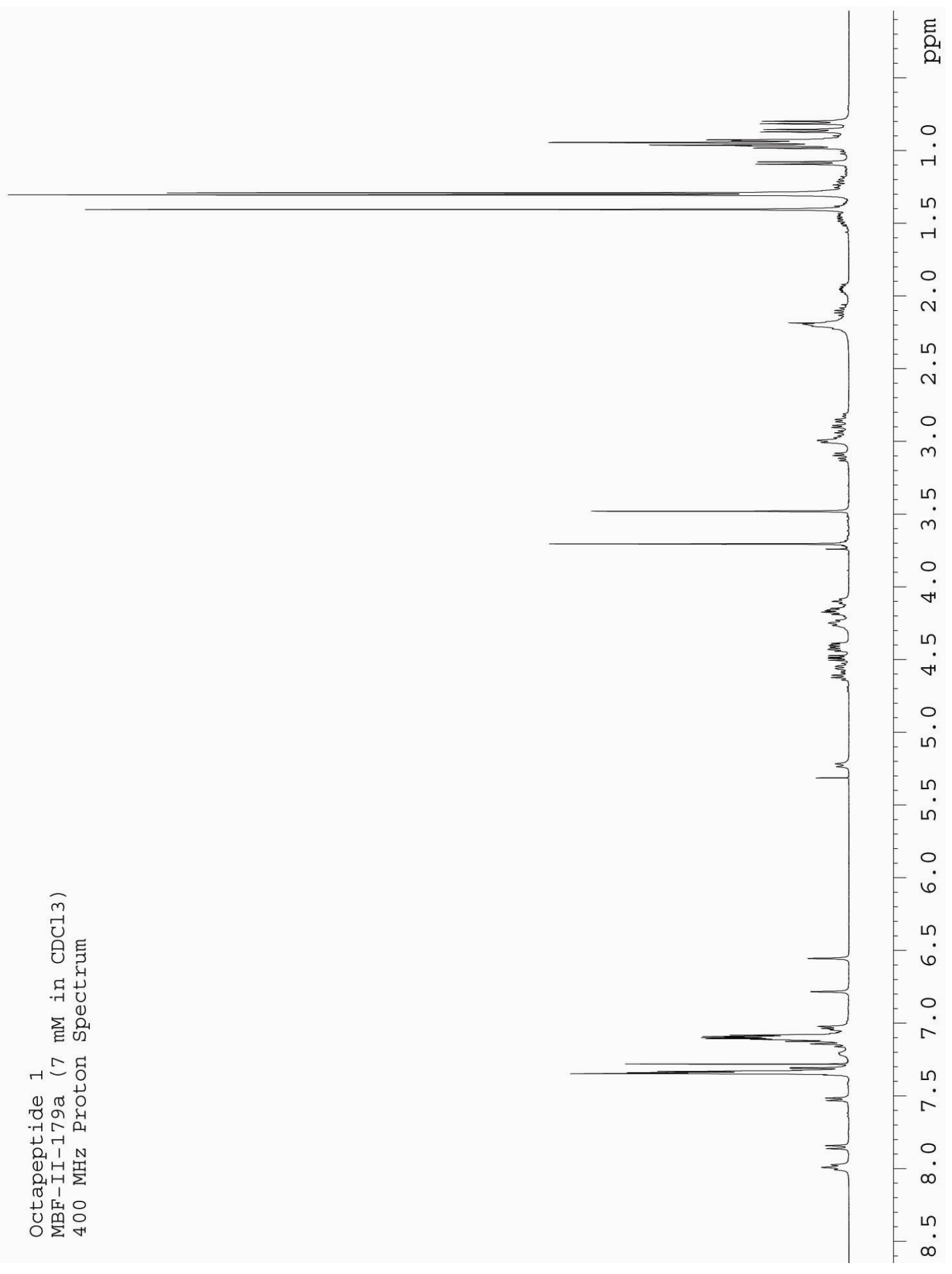

Page SI-12 
Octapeptide 1

MBF-II-179A $7 \mathrm{mM}$ in $\mathrm{CDCl} 3$

$400 \mathrm{MHz}$ 2D gs-COSY Experiment

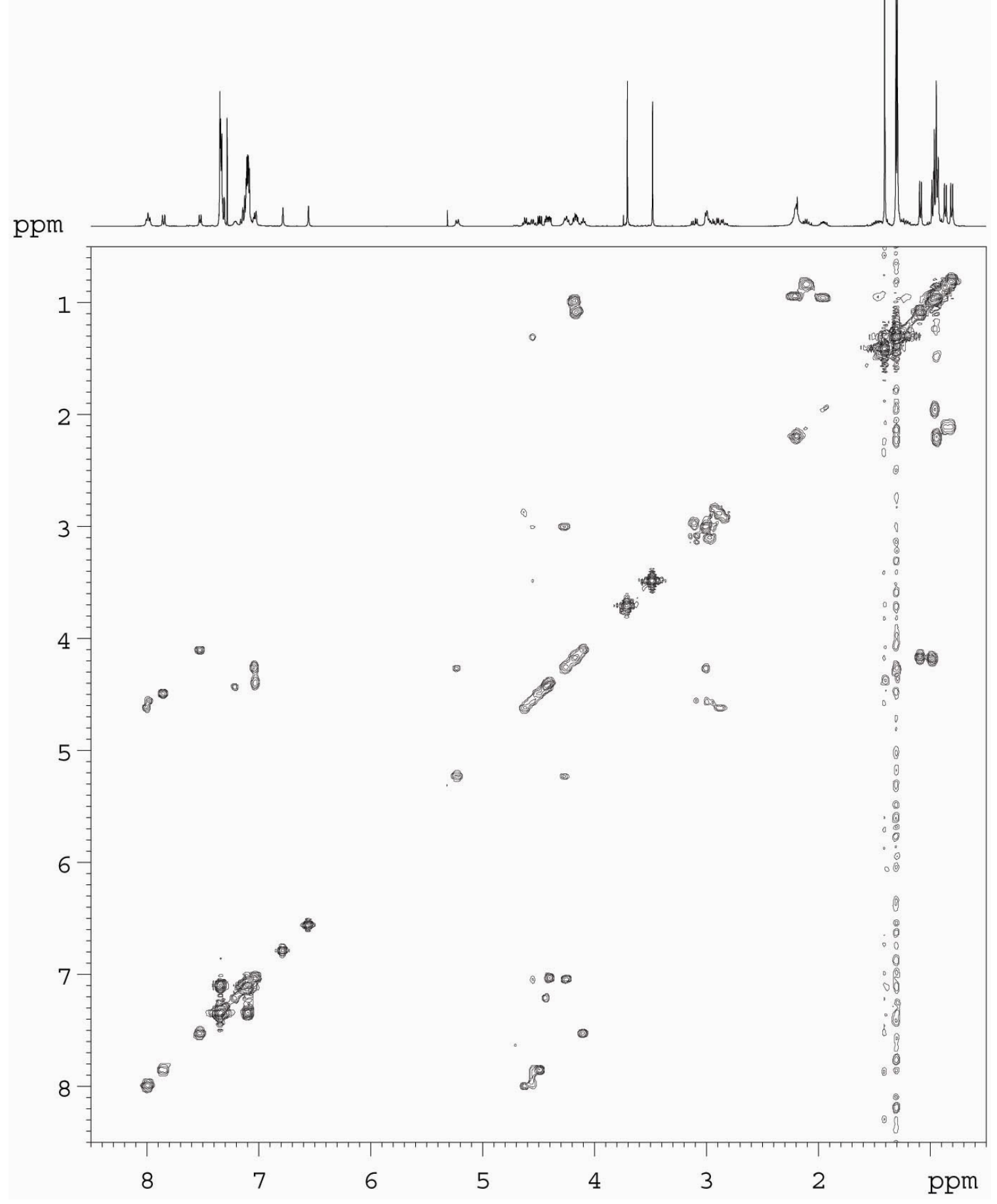

Page SI-13 
Octapeptide 1

MBF-II-179A $7 \mathrm{mM}$ in CDCl3

$400 \mathrm{MHz} 2 \mathrm{D}$ TOCSY Experiment
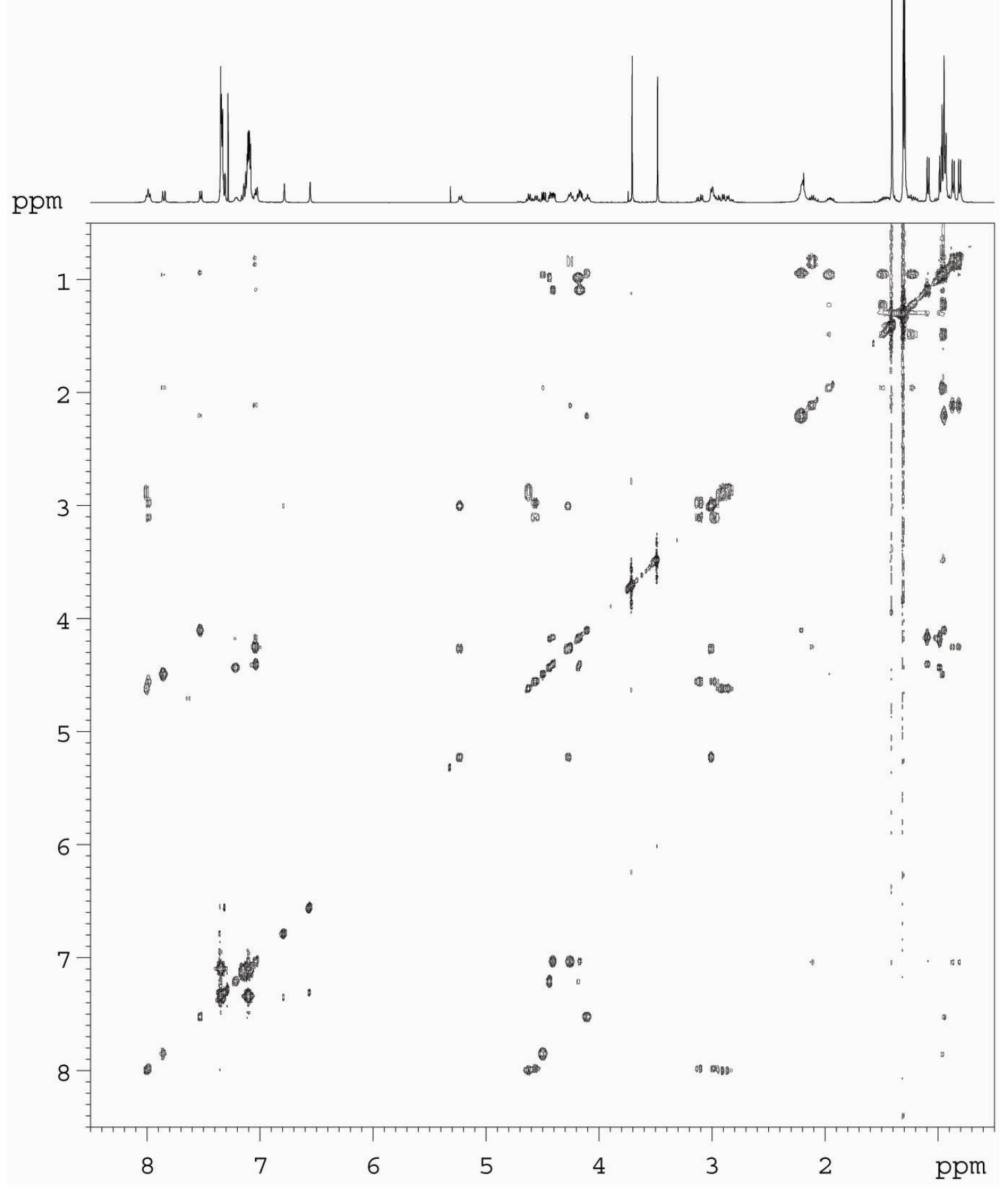

Page SI-14 
Octapeptide 1

MBF-II-179A $7 \mathrm{mM}$ in $\mathrm{CDCl} 3$

$400 \mathrm{MHz}$ 2D ROESY Experiment, $600 \mathrm{~ms}$ spin lock

Red: negative contours

Black: positive contours
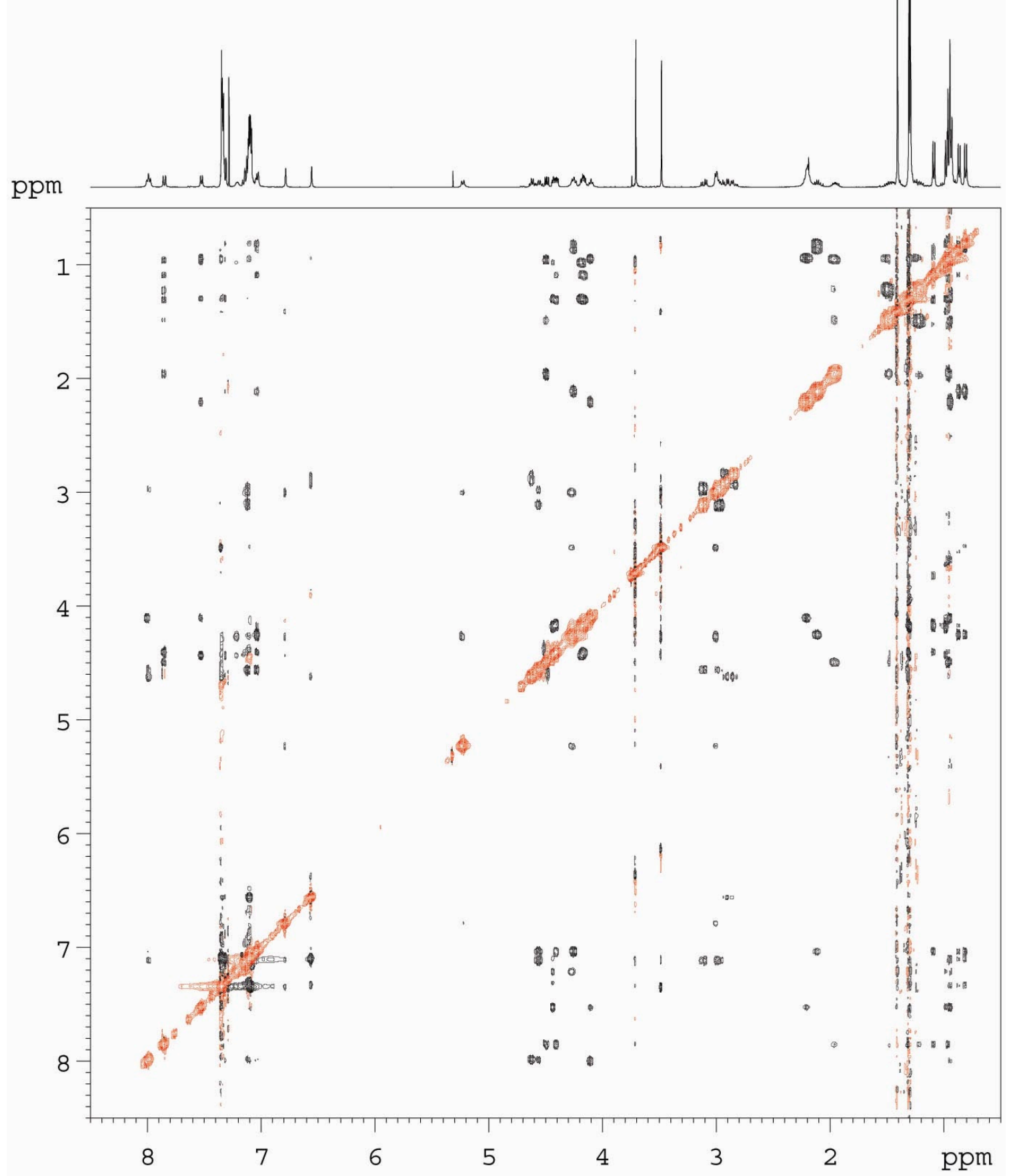

Page SI-15 


\section{References and Notes}

[1] Kagan, H.B.; Fiaud , J.C., Top. Stereochem. 1988, 18, 249-330.

[2] Copeland, G.T.; Miller, S.J., J. Am. Chem. Soc. 2001, 123, 6496-6502.

[3] Novabiochem, cat\# 01-64-0122.

[4] Bodanszky, M.; Bodanszky, A., The Practice of Peptide Synthesis; Springer-Verlag: Berlin, 1993.

[5] See reference 2.

[6] See reference 2. 